Research Paper

\title{
Activation of Central Alpha 7 Nicotinic Acetylcholine Receptor Reverses Suppressed Immune Function of T Lymphocytes and Protects Against Sepsis Lethality
}

\author{
Chao Ren ${ }^{1,2}$, Xiu-hua Li1 ${ }^{13}$, Shi-bin Wang ${ }^{1}$, Li-xue Wang1, Ning Dong1, Yao Wu ${ }^{1}$, Yong-ming Yao ${ }^{1,2,4} \bowtie$ \\ 1. Trauma Research Center, First Hospital Affiliated to the Chinese PLA General Hospital, Beijing 100048, People's Republic of China. \\ 2. School of Medicine, Nankai University, Tianjin 300071, People's Republic of China. \\ 3. Emergency Department, Jin Hua Municipal Central Hospital, Jinhua 321001, People's Republic of China. \\ 4. State Key Laboratory of Kidney Disease, the Chinese PLA General Hospital, Beijing 100853, People's Republic of China. \\ Chao Ren, Xiu-hua Li, and Shi-bin Wang have contributed equally to this work. \\ $\square$ Corresponding author: Yong-Ming Yao, MD, PhD, Trauma Research Center, First Hospital Affiliated to the Chinese PLA General Hospital, 51 Fucheng Road, \\ Haidian District, Beijing 100048, People's Republic of China. Tel: (+86)1066867394; Fax: (+86)1068989955; E-mail: c_ff@sina.com \\ (c) Ivyspring International Publisher. This is an open access article distributed under the terms of the Creative Commons Attribution (CC BY-NC) license \\ (https://creativecommons.org/licenses/by-nc/4.0/). See http://ivyspring.com/terms for full terms and conditions.
}

Received: 2017.12.27; Accepted: 2018.04.07; Published: 2018.05.12

\begin{abstract}
Sepsis remains the leading cause of high mortality and huge financial burden in intensive care units (ICU), but with scarce effective treatments due to refractory multiple organ dysfunction and persistent immunosuppression. Treatments that aim at modulating immune function and attenuating multiple organ injury will certainly benefit septic cases. Alpha 7 nicotinic acetylcholine receptor $(\alpha 7 n A c h R)$ has been reported with potent immunomodulatory properties in various diseases as the essential mediator of the cholinergic anti-inflammatory pathway (CAP). Few studies have demonstrated the potential effect of central $\alpha 7 n A c h R$ on the progression and prognosis of septic response, while its expression was first discovered on neurons and most abundant in the central nervous system. In the present study, it was found severe damage of multiple organs under the operation of cecal ligation and puncture (CLP) in rats, including heart, liver, kidneys, and lungs, as evidenced by abnormal histomorphology and notable elevation of injury markers. Concurrently, the function of spleen $\mathrm{CD}^{+} \mathrm{T}$ cells was disrupted under septic challenge, accompanied by polarization of helper $\mathrm{T}$ cell (Th)2, which exhibited outward signs of immunosuppression. Intracerebroventricular injection of PNU282987, a selective agonist of $\alpha 7 \mathrm{nAchR}$, significantly alleviated multiple organ injury, reversed immunosuppressive state, and improved the outcome of septic rats, while they were exacerbated by treatment with methyllycaconitine, a selective antagonist of $\alpha 7 n A c h R$. This study provides the first evidence that activation of central $\alpha 7 \mathrm{nAchR}$ is beneficial for attenuating multiple organ dysfunction as well as abnormal immune response, and improving the prognosis of rats when exposed with sepsis.
\end{abstract}

Key words: Alpha 7 nicotinic acetylcholine receptor; sepsis; immune response; multiple organ dysfunction syndrome; prognosis

\section{Introduction}

Sepsis is recognized as a major cause of high mortality rate and huge financial burden in intensive care units (ICU), and characterized by refractory multiple organ dysfunction syndrome (MODS) and recurrent infection due to dysregulated response to infection. Although the interaction between invaded pathogens and host immune system is the initial step for sepsis, the balance between pro- and anti-inflammatory response determines the fate of septic patient [1]. Therefore, maintaining dynamic homeostasis of immune response is the prerequisite for improving the prognosis of septic patients. Current studies have shown that more than $75 \%$ of deaths in sepsis are patients over the age of 65 due to 
immunosenescence and increased comorbidities [2]. Immunosuppression, as a result of reprogramming of immune cells, was considered as a major abnormality that drove sepsis toward a highly lethal condition [3]. Mice that underwent cecal ligation and puncture (CLP) operation developed remarkable signs of immunosuppression on day one, including inadequate response of immune cells and high bacterial load, followed by poor outcomes [4]. Clinical data also provided a close relationship between immunosuppressive state and unfavorable outcome of septic patients [5]. Early recognition and treatments that target abnormal immune response and organ dysfunction might be of great benefits to against lethal sepsis.

Alpha 7 nicotinic acetylcholine receptor ( $\alpha 7 n A c h R)$ is an essential mechanism for the cholinergic anti-inflammatory pathway (CAP) which has revealed potent immunomodulatory properties in various diseases after proposed by Tracey in 2002 [6]. Multiple drugs that targeted peripheral a7nAchR significantly improved survival rates of septic animals by reducing production of inflammatory mediators and restoring function of immune cells [7, 8]. For example, GTS-21, a selective agonist of a7nAchR, dose-dependently down-regulated serum tumor necrosis factor- $\alpha$ (TNF- $\alpha$ ) and high mobility group box-1 protein (HMGB1) levels in septic mice, accompanied with improved survival rates, indicating that peripheral $a 7 n A c h R$ might be a potential therapeutic target for sepsis [7]. Up to now, few studies have focused on exploring the potential effect of central $\alpha 7 \mathrm{nAchR}$ on the progression and prognosis of septic challenge, while its expression was first discovered on neurons and most abundant in the central nervous system [9]. Actually, the central a7nAchR has been identified as responsible for cognition, memory and sensory processing after discovered localization in various brain regions, including cortex, hippocampus, subcortical limbic regions and brainstem nuclei [10]. Further researchers have found that the expression of neuronal a7nAchR was detected at both pre- and post-synaptic loci, and implicated in neurotransmitter release and signaling transmission for learning and memory [11]. Interestingly, activation of brain $a 7 n A c h R$ was reportedly shown to elevate both acetylcholine and dopamine concentrations, representatives for two distinct neural pathways, although it was considered the primary receptor for cholinergic system [12]. This might be because choline and dopamine were both needed for regulating neurological function. Activation of brain a7nAchR did improve cognition, attention, and memory of mice subjected to neurological impairment [13]. Alzheimer's disease, as an example, was reportedly prevented by a7nAchR agonists through reducing neurotoxicity of $A \beta$ peptide, down-regulating neuro-inflammation, and enhancing neurite outgrowth [14]. While animals with central a7nAchR inhibited or gene knockdown presented with deteriorating cognitive and memory impairment in multiple neurological disorders, such as schizophrenia, autism, and intracerebral hemorrhage [10, 15]. Therefore, central a7nAchR might be a promising drug target to improve brain injury diseases as one of the major resolution mechanisms. At present, the system of neuroendocrine-immune network has provided a new horizon for sepsis treatments, especially the CAP that showed great benefits for septic animals by controlling inflammation and restoring immune function [16]. Additionally, it might show a more economical and efficient way to modulate inflammatory response and presents with novel therapeutic targets for sepsis. Thus, the aim of this study was to investigate the effects of central a7nAchR on multiple organ dysfunction as well as peripheral immune response, and the outcome of rats following sepsis.

\section{Materials and Methods}

\section{Animals}

Male SD rats (weighing $250 \pm 20$ g) were purchased from Laboratory Animal Science of Chinese Academy of Medical Sciences, Beijing, China. All rats were kept in air-conditioned room with 12 hours (h) light and $12 \mathrm{~h}$ dark cycle, and free access to forage and water. All experiments were conducted in accordance with the National Institutes of Health (NIH) Guidelines and approved by the Scientific Investigation Board of the Chinese PLA General Hospital (No. SYXK2012-0014), Beijing, China.

\section{Canal insertion of lateral ventricles}

Rats were anesthetized by inhaling isoflurane (5\% induction, $3 \%$ maintenance), and placed on the motorized stereotactic apparatus (Stoelting Co., Wood Dale, IL) with thermal pad to maintain body's temperature. The scalp of rat was disinfected by $10 \%$ povidone-iodine after hair shaved. A 2-cm-long middle incision was made to expose skull and bregma that was set as coordinate zero $(x=0, y=0, z=0)$. Then the catheter was inserted into the left lateral ventricle and fixed by acrylic dental cement after a $1.0 \mathrm{~mm}$ diameter craniotomy was made in predetermined coordinates $(x=-0.72 \mathrm{~mm}, \mathrm{y}=2.0 \mathrm{~mm}, \mathrm{z}=0)$. Rats were rested for at least 7 days before another operation.

\section{Cecal ligation and puncture(CLP)}

CLP operation was used to reproduce septic 
model. Briefly, rats were anesthetized by intraperitoneal injection of chloral hydrate $(5 \%, 30$ $\mathrm{mg} / \mathrm{kg}$ of body weight), and laid on the operating table. A 2-cm-long middle abdominal incision was made to expose the cecum after skin disinfection. The cecum was ligated at $75 \%$ distance between distal pole and the base of the cecum, and punctured once with 16-gauge needle. A small amount of feces was extruded by compressing the ligated cecum slightly. Then, the abdominal incision was closed in the wake of relocation of the exposed cecum. All rats were subcutaneously injected normal saline $(5 \mathrm{ml}$ per $100 \mathrm{~g}$ body weight) for fluid resuscitation. The sham group rats had cecum exposed without ligating and puncturing.

\section{Intracerebroventricular (ICV) injection}

Rats were randomly divided into three groups: the sham group, the sepsis group, and the sepsis with pharmacological intervention group. In the sepsis with pharmacological intervention group, PNU-282987 (Sigma, St. Louis, MO, with doses at 0.25 $\mu \mathrm{g}, 0.8 \mu \mathrm{g}$, and $2.56 \mu \mathrm{g}$ ), a selective agonist of a7nAchR, and methyllycaconitine (MLA, Tocris, UK, with doses at $6 \mathrm{ng}, 12 \mathrm{ng}$, and $24 \mathrm{ng}$ ), a selective antagonist of $\alpha 7 n A c h R$, were injected into the lateral ventricle at $0 \mathrm{~h}, 6 \mathrm{~h}$ after CLP surgery, respectively. The ICV injection was conducted in rats anesthetized by inhaling isoflurane. Drugs that dissolved in saline solution were administrated in a volume of $5 \mu \mathrm{l}$ through a pre-assembled syringe needle. Then, the syringe was removed at $5 \mathrm{~min}$ after injection to avoid backflow. Rats of the sham group were given the same volume of normal saline. The survival rates of rats were recorded at $12 \mathrm{~h}, 24 \mathrm{~h}, 36 \mathrm{~h}, 48 \mathrm{~h}, 72 \mathrm{~h}, 96 \mathrm{~h}$, $120 \mathrm{~h}, 144 \mathrm{~h}$, and $168 \mathrm{~h}$ post CLP surgery.

\section{Histological assessment}

Specimens of heart, liver, lungs, and kidneys were harvested and fixed in $4 \%$ paraformaldehyde. These tissues were further dehydrated by ethanol gradient and embedding with paraffin. The paraffin-embedded samples were cut into 5 - $\mu \mathrm{m}$ thick slices for hematoxylin-eosin (HE) staining. Histological alterations were imaged and analyzed by light microscope.

\section{Measurements of organ functional parameters}

Serum samples were collected through the carotid artery, and centrifuged $(1500 \mathrm{~g}, 15 \mathrm{~min})$ to obtain the serum that was further used to analyze the biochemical parameters of heart, liver and renal function, including creatine kinase $(\mathrm{CK})$, creatine kinase-MB (CK-MB), aspartate aminotransferase (AST), alanine aminotransferase (ALT), cholyglycine (CG), blood urea nitrogen (BUN), and creatinine (Cr).
All these biochemical parameters were analyzed via HITACHI7600 (Hitachi, Tokyo, Japan). The wet-to-dry weight ratio (W/D) and myeloperoxidase (MPO) activity in pulmonary tissues were quantified as indicators for lung injury. The left lung was separated after rat was killed by overdose anesthetic, and its wet weight was recorded without blood and other contaminants. Then, the lung was dried at $80^{\circ} \mathrm{C}$ for $24 \mathrm{~h}$ to measure the dry weight and calculate the W/D. MPO activity was assessed by enzyme-linked immunosorbent assay (ELISA, Hycult Biotech, Netherlands) according to the manufacturer's instruction after lung tissues were homogenized and quantified.

\section{Isolation of splenic T lymphocytes}

Splenocytes were harvested through a 300s stainless steel mesh after extraction and dispersion of rat spleens. Mononuclear cells were obtained by Ficoll-Paque density gradient centrifugation (3000 $\mathrm{rpm}, 15 \mathrm{~min})$. The $\mathrm{CD}^{+} \mathrm{T}$ cells were then isolated from splenic mononuclear cells by magnetic cell sorting system (Miltenyi Biotech, Bergisch Gladbach, Germany) in accordance with the manufacturer's instructions. Splenic mononuclear cells were incubated with anti-CD4 magnetic microbeads $(20 \mu \mathrm{l}$ per $10^{7}$ cells) at $4^{\circ} \mathrm{C}$ for $15 \mathrm{~min}$. Then, the $\mathrm{CD} 4^{+} \mathrm{T}$ lymphocytes were collected after magnetic separation with MS columns.

\section{Cell counting kit-8 (CCK8) assay}

The proliferation of splenic $\mathrm{CD}^{+} \mathrm{T}$ cells was measured by CCK8 kit (Dojindo Laboratories, Kumamoto, Japan), according to the manufacturer's instructions. Purified $\mathrm{CD}^{+} \mathrm{T}$ cells that were resuspended in complete RPMI 1640 medium supplemented with $10 \%$ fetal calf serum, $100 \mathrm{U} / \mathrm{ml}$ penicillin, and $100 \mu \mathrm{g} / \mathrm{ml}$ streptomycin were incubated with concanavalin A (Con A, $5 \mu \mathrm{g} / \mathrm{ml}$, Sigma, St. Louis, $\mathrm{MO}$ ) in a humidified incubator $\left(37^{\circ} \mathrm{C}, 5 \% \mathrm{CO}_{2}\right)$ for $68-72 \mathrm{~h}$. Then, $10 \mu \mathrm{l} \mathrm{CCK} 8$ solution was added into the cell suspension $\left(100 \mu \mathrm{l}, 2 \times 10^{6}\right.$ cells $/ \mathrm{ml}$ ), and further incubated for $2 \mathrm{~h}$. The optical density value was measured at $450 \mathrm{~nm}$ by enzyme-linked immunosorbent assay plate reader (Spectra MR, Dynex, Richfield, MN).

\section{Enzyme-linked immunosorbent assay}

Levels of interleukin-2 (IL-2), IL-4, and interferon- $\gamma$ (IFN- $\gamma)$ in culture supernatants were all quantified by ELISA kits (Excell Inc., Shanghai, China), complying with the protocols provided by manufacturers. The results were recorded at $450 \mathrm{~nm}$ by enzyme-linked immunosorbent assay plate reader (Spectra MR, Dynex, Richfield, MN). 


\section{Quantitative real-time polymerase chain reaction (PCR)}

The mRNA expressions of IL-2, IL-4, and IFN- $\gamma$ in $\mathrm{CD}^{+} \mathrm{T}$ cells were quantified by quantitative real-time PCR. Total RNA was extracted from purified $\mathrm{CD}^{+} \mathrm{T}$ cells by Trizol reagent (Invitrogen, California, CA) following the manufacturer's instruction. The cDNA was synthesized from total RNA through reverse transcription system (Promega, Madison, WI). The cDNA was prepared in triplicate, and quantified using SYBR Green PCR Master Mix, compounding following primers: rat IL-2, 5'-CAG CGTGTGTTGGATTTGAC-3' (forward) and 5'-TGAT GCTTTGACAGATG-GCTA-3' (reverse); rat IL-4, 5'-AACAAGTCTGGGGTTCTCGG-3' (forward) and 5'-TGTTGTGAGCGTGGACTCAT-3' (reverse); rat IFN- $\gamma, 5^{\prime}$-AGGTGAACAACCCACAGAT-3' (forward) and 5'-CTTCTTATTGGCACACTCTCTAC-3' (reverse). The gene expression was normalized with $\beta$-actin, and evaluated by the equation $2^{-\mathrm{dCt}}$.

\section{Statistical analysis}

All data was analyzed by SPSS 19.0, and expressed as mean \pm standard deviation (SD) of more than three independent experiments performed in triplicate. One-way analysis of variance (ANOVA) was needed for evaluating differences among multiple groups, and student $t$-test was used to assess significant differences for intergroup. Survival were analyzed by Kaplan-Meier survival curves, and compared using the log rank test. $P$ values $<0.05$ were considered as statistically significant.

\section{Results}

\section{ICV injection of PNU282987 ameliorated sepsis induced multiple organ injury}

Morphological changes of heart, liver, lungs, and kidneys were assessed at $24 \mathrm{~h}$ after CLP surgery, and presented direct evidences of tissue injury, as shown in Fig. 1A. The pathological examination of rat heart in the sepsis group revealed remarkable infiltration of inflammatory cells, focal myocardial cell edema, and necrosis in comparison with the sham group which showed clear structure and shape of myocardial cells (Fig. 1A, heart). We further quantified levels of serum CK (creatine kinase) and CK-MB (creatine kinase-MB), specific markers for myocardial damage, and found that both indicators displayed higher levels in the sepsis group than those in the sham group (Fig. 1B), indicating a severely damaged heart under septic challenge. The activation of brain $a 7 n A c h R$ was conducted by ICV injection of PNU282987 as mentioned above, and we noticed that treatment with PNU282987 significantly ameliorated sepsis induced heart injury, as evidenced by improved morphology and structure of myocardial cells (Fig. 1A), and declined levels of serum CK and CK-MB (Fig. 1B) when compared to the sepsis groups, but without dose-dependent effects.

The pathological features of liver also displayed obvious differences between the sepsis and the sham groups. The liver tissues of septic rats exhibited disordered arrangement of hepatocytes, focal congestion and dilatation, and elevated infiltration of lymphocytes, but no marked abnormalities were seen in liver of the sham group (Fig. 1A). The serum makers of hepatic injury, including AST, ALT, and CG, were all presented with prominent elevations in the sepsis groups when compared with the sham groups, while these effects were significantly reduced by ICV injection of PNU282987 (Fig. 1C).

The pathological sections of lungs under sepsis exposure were presented in Fig. 1, which revealed acute lung injury, including disorganized alveolar structures, expanded interstitium due to edema and inflammatory infiltrations, and focal congestion and hemorrhage of alveoli, while no remarkable lesions were found in the sham group. Wet to dry weight ratio and MPO activity in lung tissues were also specific indicators for pulmonary edema and acute lung injury, and both showed markedly elevation in the sepsis group in comparison to the sham group (Fig. 1D). Treatment with PNU282987 was identified to alleviate sepsis induced acute lung injury by attenuating morphological changes and reducing $\mathrm{W} / \mathrm{D}$ ratio and MPO levels after ICV injection (Fig. 1A, D).

The rat kidneys were vulnerable to septic exposure, and showed diffuse damage in histological assessment after the onset of sepsis. Multifocal hemorrhage and interstitial edema were the main features of rat kidneys compromised by sepsis, as shown in Fig. 1-A. Diffuse infiltration of inflammatory cells and narrowed lumens of renal tubular due to swollen and caducous epithelial cells were also observed in these pathological sections of rat kidneys. Additionally, serum BUN and $\mathrm{Cr}$ exhibited higher levels in the sepsis group than those in the sham group (Fig. 1E). Central administration of PNU282987 significantly attenuated sepsis induced acute kidney injury (Fig. 1A, E).

\section{ICV injection of MLA exacerbated multiple organ injury in septic rats}

The morphological changes and serum indicators of heart, liver, lungs, and kidneys were analyzed after central a7nAchR was inhibited by MLA injection. The pathological sections of heart revealed more severe damage in the sepsis with MLA 
injection groups than those in the sepsis groups, as evidenced with wave-like arrangement of cardiomyocytes, increased infiltration of inflammatory cells, and notable edema and necrosis of myocardial cells. However, no significant differences were seen in various doses of MLA treated groups (Fig. 2A). Levels of serum CK and CK-MB were observed higher levels in the MLA treated sepsis groups than those in the sepsis groups (Fig. 2B).
Liver in the MLA treated groups presented deteriorated impairment in histological assessment, including disappeared normal structure of liver tissues, diffusive edema and necrosis of hepatic cells, accompanied by aggravated infiltration of inflammatory cells (Fig. 2A). ICV administration of MLA enhanced the release of AST, ALT, and CG, as levels of these serum markers were much higher in the sepsis with MLA treated groups than the sepsis groups (Fig. 2C).

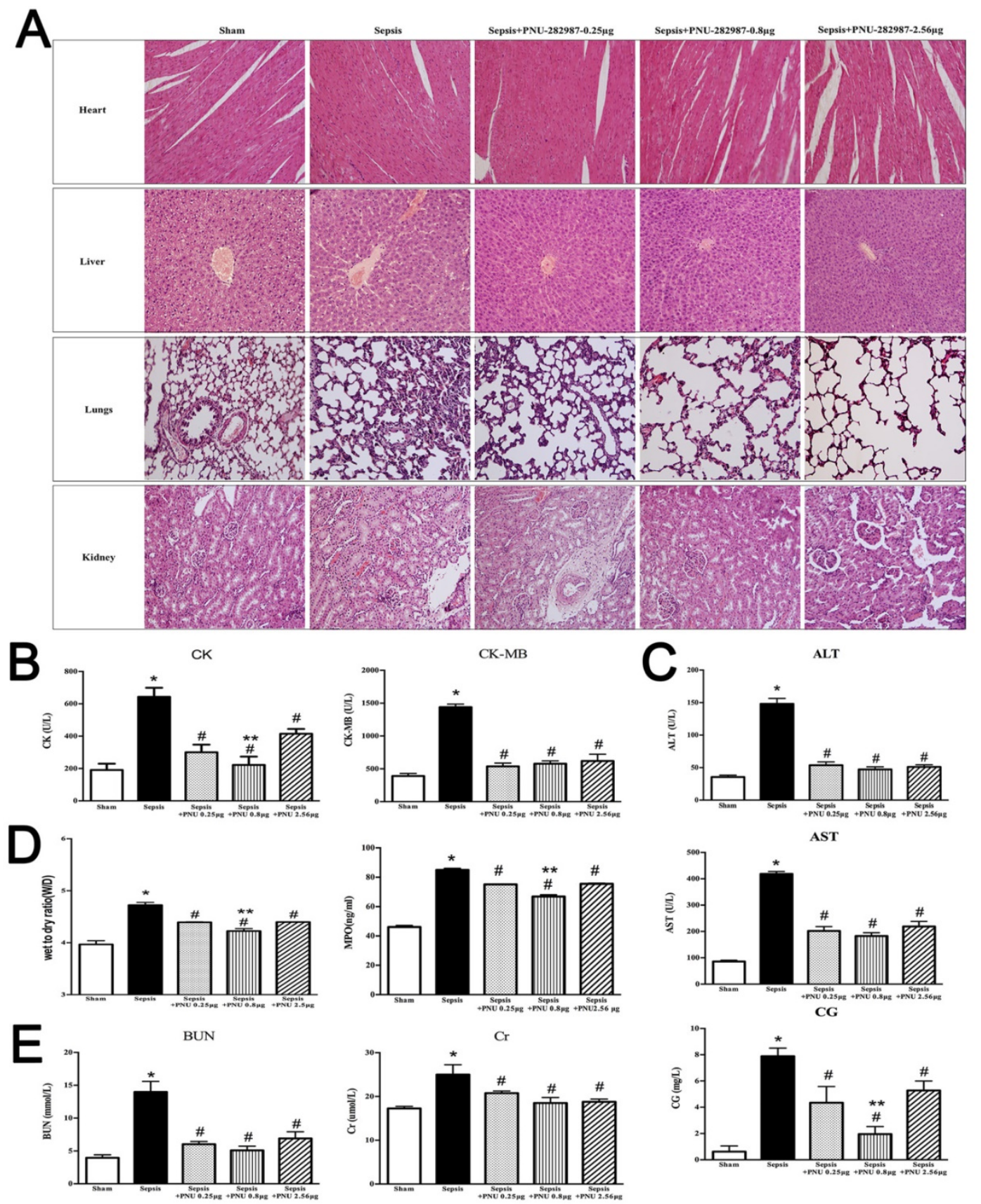

Figure 1. ICV injection of PNU282987 ameliorated sepsis induced multiple organ dysfunction. (1A) Histopathologic changes of heart, liver, lungs, and kidneys were measured by HE staining ( $\times 100$ magnification). (1B, 1C, and 1E) Serum biochemical parameters, including creatine kinase (CK), CK-MB, aspartate aminotransferase (AST), alanine aminotransferase (ALT), cholylglycine (CG), blood urea nitrogen (BUN), and creatinine (Cr), were quantified by HITACHI 7600 to reflect organ dysfunction, respectively $\left(n=6,{ }^{*} P<0.01\right.$ as compared with the sham groups; $\# P<0.01$ as compared with the sepsis groups; ${ }^{* *} P<0.01$ as compared with other dose groups). (ID) Wet to dry ratio (W/D) and myeloperoxidase (MPO) activity of pulmonary tissues were quantified by weighting and ELISA kit, respectively ( $\mathrm{n}=6$, ${ }^{*} P<0.01$ as compared with the sham groups; $\# P<0.01$ as compared with the sepsis groups; ${ }^{* *} P<0.01$ as compared with other dose groups). 


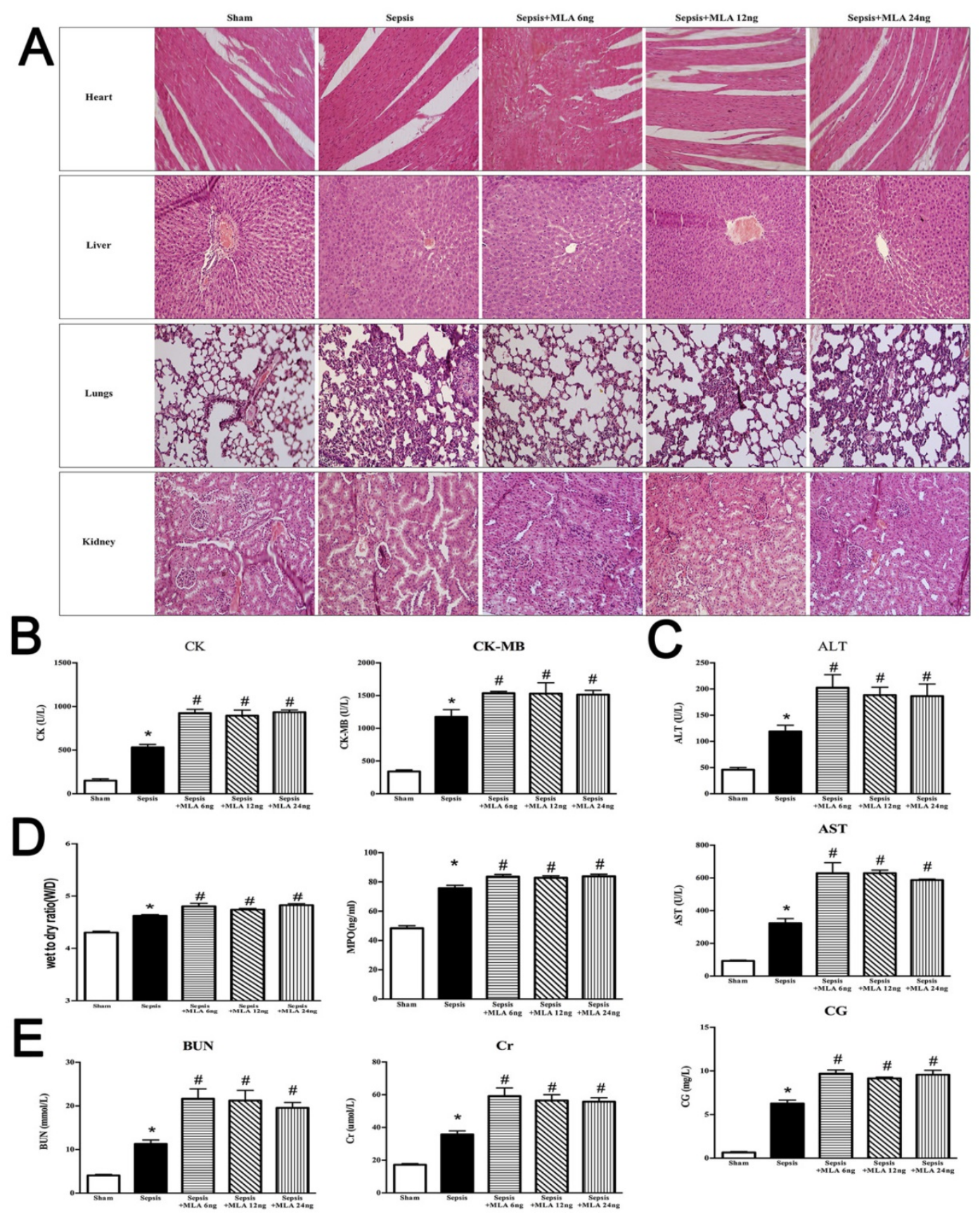

Figure 2. ICV injection of MLA exacerbated multiple organ injury in septic rats. (2A) Histopathologic alterations of heart, liver, lungs, and, kidneys were determined by HE staining ( $\times 100$ magnification). (2B, 2C, and $2 E)$ Serum biochemical parameters, including CK, CK-MB, AST, ALT, CG, BUN, and Cr, were quantified by HITACHI 7600 to reflect organ dysfunction, respectively $\left(n=6,{ }^{*} P<0.01\right.$ as compared with the sham groups; $\# P<0.01$ as compared with the sepsis groups). (2D) W/D ratio and MPO activity of pulmonary tissues were quantified by weighting and ELISA kit, respectively $\left(n=6,{ }^{*} P<0.01\right.$ as compared with the sham groups; $\# P<0.01$ as compared with the sepsis groups).

It was shown that lungs underwent more serious injury in the sepsis with MLA injection group when compared to the sepsis group. Pathological sections revealed more infiltration of inflammatory cells, diffusive hemorrhage and congestion of alveoli, and increased thickness of interstitium in the MLA treated groups, accompanied with elevated $\mathrm{W} / \mathrm{D}$ ratio and MPO activity when compared to the sepsis groups (Fig. 2).
ICV injection of MLA markedly exacerbated acute kidney injury under septic challenge, as shown in Fig. 2. More infiltrated inflammatory cells and collapsed epithelial cells were observed in kidneys of the MLA treated groups. Meanwhile, disorganized structure was a main feature of exacerbated acute kidney injury in the treatment with MLA, along with elevated serum BUN and Cr levels. 


\section{The time-dependent effects of treatment with PNU282987 and MLA on sepsis induced multiple organ dysfunction}

In order to maximize the intelligence of the effects of central $a 7 n A c h R$ on sepsis induced multiple organ dysfunction, we evaluated the time-dependent response of PNU282987 and MLA at 24 h, 48 h, and 72 $h$ post CLP operation. The doses that functioned with maximum impact in the dose-dependent experiments of PNU282987 and MLA were selected respectively (PNU282987: $0.8 \mu \mathrm{g}$ and MLA: $12 \mathrm{ng}$ ). The function of heart, liver, lungs, and kidneys were measured by injury parameters, including levels of $\mathrm{CK}, \mathrm{CK}-\mathrm{MB}$, AST, ALT, CG, BUN, Cr, MPO, and W/D ratio (Fig. 3). ICV injection of PNU282987 did improve the function of heart, liver, lungs, and kidneys at different time points, while exacerbated effects were seen by ICV injecting MLA, as shown in Fig. 3.

\section{The effects of treatment with PNU282987 on proliferation and secretion of splenic $\mathrm{CD4}^{+} \mathrm{T}$ cells in sepsis}

In the current study, the proliferative activity of $\mathrm{CD}^{+} \mathrm{T}$ cells was determined by CCK8 assay. In the sepsis group, the proliferation of $\mathrm{CD}^{+} \mathrm{T}$ cells was suppressed in comparison to the sham group (Fig. 4A). IL-2, IL-4, and IFN-y, secreted by CD4+ ${ }^{+}$cells, were major representatives for the function and polarization of $\mathrm{CD}^{+} \mathrm{T}$ cells. Concentrations of IL-2 and IFN- $\gamma$ in culture supernatants were both strikingly reduced in the sepsis groups when compared to the sham groups. However, IL-4 concentration revealed a marked increase in the sepsis group (Fig. 4B). We further quantified the mRNA expressions of IL-2, IL-4, and IFN- $\gamma$ in $\mathrm{CD}^{+} \mathrm{T}$ cells by quantitative real-time PCR. The results of gene expressions were in line with the trends of protein expressions (Fig. 4C). ICV injection of PNU282987 significantly reversed the suppressed proliferation and function of $\mathrm{CD}^{+} \mathrm{T}$ cells in the setting of sepsis, as evidenced by enhanced proliferation, increased expressions and release of IL-2 as well as IFN- $\gamma$, and inhibited production of IL-4 when compared to the sepsis groups. In addition, PNU282987 was in maximum effect when dosed at $0.8 \mu \mathrm{g}$ (Fig. 4).

\section{ICV injection of MLA exacerbated dysfunction of splenic $\mathrm{CD4}^{+} \mathrm{T}$ cells in septic rats}

ICV injection of MLA showed a potent suppressive effect on splenic $\mathrm{CD} 4^{+} \mathrm{T}$ cells, manifested by inhibited proliferative activity, reduced expressions and release of IL-2 and IFN- $\gamma$, but elevated IL-4 levels in compared to the sepsis group, which displayed an exacerbated immunosuppressive state compromised by sepsis (Fig. 5).
The time-dependent effects of treatment with PNU282987 and MLA on immune function of $\mathrm{CD4}^{+} \mathrm{T}$ cells in sepsis

The splenic $\mathrm{CD}^{+} \mathrm{T}$ cells were harvested at $24 \mathrm{~h}$, $48 \mathrm{~h}$, and $72 \mathrm{~h}$ post CLP operation. The CD4 ${ }^{+} \mathrm{T}$ cells presented with remarkable disturbance in proliferation over time after sepsis initiation (Fig. 6A). Likely, productions of IL-2 and IFN- $\gamma$ were significantly inhibited at $48 \mathrm{~h}$ and $72 \mathrm{~h}$ after the onset of sepsis, indicating a time-dependent suppressive state of $\mathrm{CD}^{+} \mathrm{T}$ cells (Fig. 6B). IL-4 concentrations, however, remained at high levels over time post CLP surgery (Fig. 6B). The gene expressions were in conformed to the trends of protein expressions (Fig. 6C). The doses used in the present experiments were selected according to dose-dependent pattern (PNU282987: 0.8 $\mu \mathrm{g}$ and MLA: $12 \mathrm{ng}$ ). ICV injection of PNU282987 showed marked improvements in alleviating suppressed function of $\mathrm{CD} 4^{+} \mathrm{T}$ cells, as evidenced by restored proliferation, elevated release of IL-2 and IFN- $\gamma$, and reduced IL-4 expression when compared to corresponding septic groups. While treatment with MLA deteriorated the suppressed proliferation and immune dysfunction of $\mathrm{CD}^{+} \mathrm{T}$ cells induced by sepsis at various time points (Fig. 6).

\section{Effects of PNU282987 and MLA on survival rates of septic rats}

The survival rates of septic animals were recorded at $12 \mathrm{~h}, 24 \mathrm{~h}, 36 \mathrm{~h}, 48 \mathrm{~h}, 72 \mathrm{~h}, 96 \mathrm{~h}, 120 \mathrm{~h}, 144$ $h$, and $168 \mathrm{~h}$ post CLP surgery, respectively. No rats died in the sham group (survival rate: $100 \%$ ). The survival rate was $40 \%$ in the sepsis group $(n=26)$. ICV injection of PNU282987 significantly increased survival rate of septic rats in comparison to that in the sepsis group ( $\mathrm{n}=26,80 \%$ vs. $40 \%, P<0.05)$. The survival rate was $36 \%$ in the MLA treated group, and showed no remarkable difference when compared to the sepsis group (Fig. 7).

\section{Discussion}

Recently, the updated definition of sepsis that published by the third sepsis consensus conference provides new clinical criteria for early recognition and prognostic evaluation of septic patients [17]. The life threatening organ dysfunction and refractory immunosuppression are recognized as major lethal factors for septic patients $[17,18]$. Treatments that aim at modulating immune function and attenuating organ injury will certainly benefit septic patients, especially after the failed trials via antagonizing pro-inflammatory mediators [19]. CAP is one of the specified applications of neuroendocrine-immune mechanism, and it provides a new horizon for immunomodulation as its protective effect has been 
documented in various diseases [16, 20]. a7nAchR, the unique periphery receptor for $\mathrm{CAP}$, is considered as a promising therapeutic target when it is difficult to regulate the activity of systematic neuronal network [21]. Indeed, activation of $a 7 n A c h R$ has presented with significant protective effects in septic challenge, owing to its anti-inflammatory and immunoregulatory capacities [7, 22]. However, the clinical evidences of $a 7 n A c h R$ remain scarce in the treatment of septic patients, accompanied with major findings on cells and animals. One possible reason is that we can't ignore the potential role of central $a 7 n A c h R$ in immunomodulation, as it shows abundant expression in the central nervous system, and exhibits neuro-protective effect by abating local inflammation $[23,24]$.
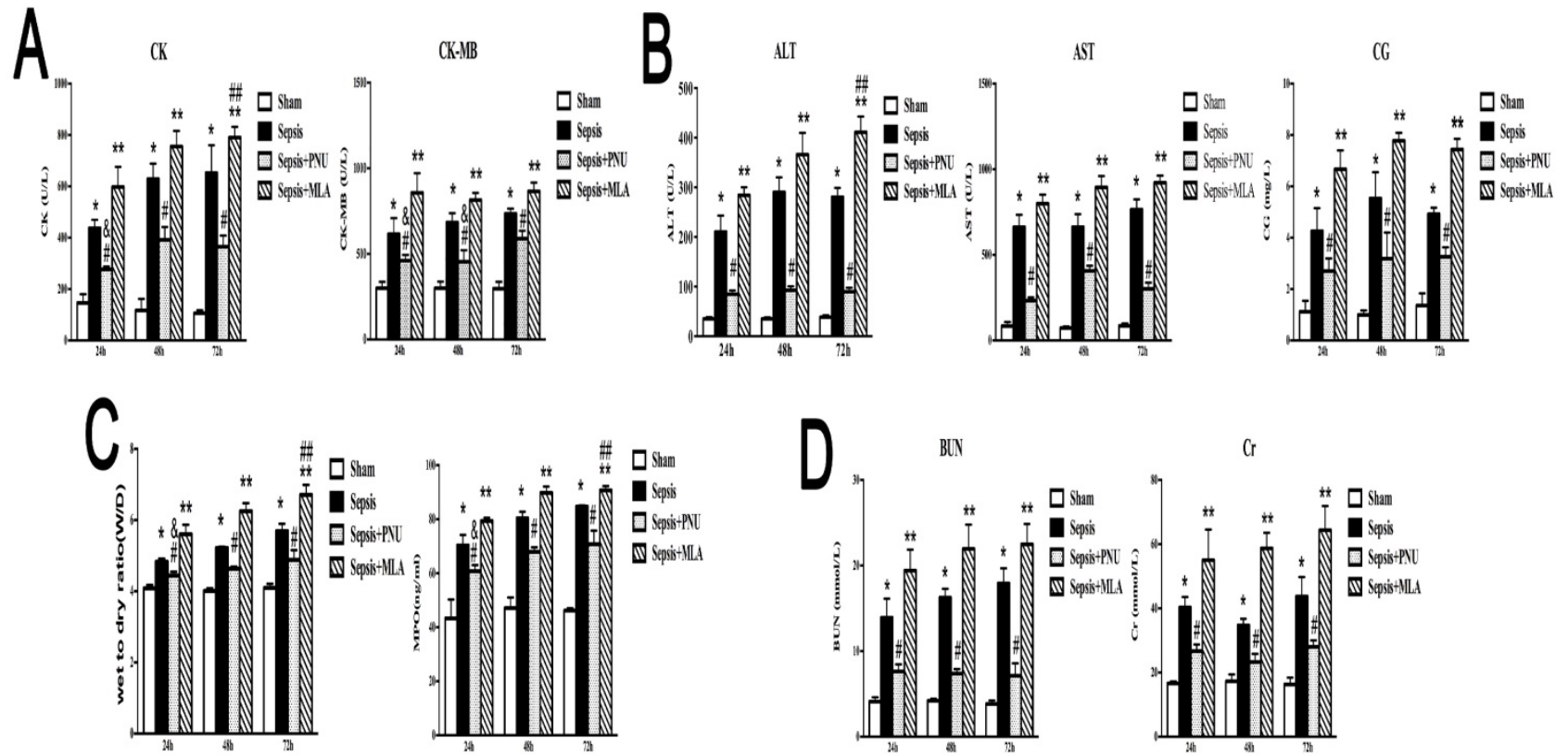

Figure 3. The time-dependent response of PNU282987 and MLA on sepsis induced multiple organ dysfunction. (3A, 3B, and 3D) Serum biochemical parameters, including CK, CK-MB, AST, ALT, CG, BUN, and Cr, were quantified by HITACHI 7600 to reflect organ dysfunction, respectively. (3C) W/D ratio and MPO activity of pulmonary tissues were quantified by weighting and ELISA kit, respectively $\left(n=6,{ }^{*} P<0.01\right.$ as compared with the sham groups at the identical time points; $\# P<0.01$ as compared with the sepsis groups at the identical time points; ${ }^{* *} P<0.01$ as compared with sepsis groups at the identical time points; $\& P<0.01$ as compared with different time points with PNU282987 administration; $\# P<0.01$ as compared with different time points with MLA administration).
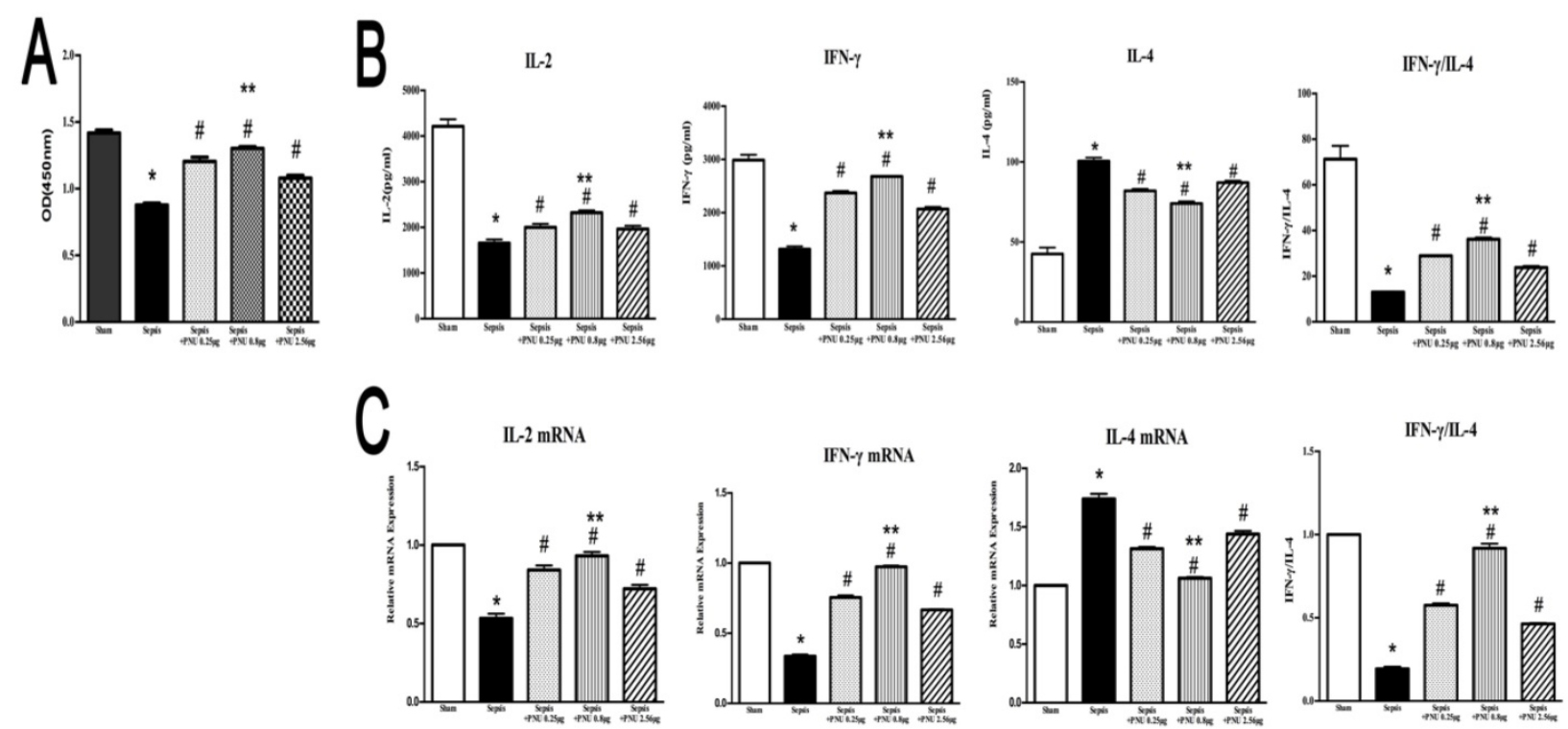

Figure 4. Effects of treatment with PNU282987 on proliferation and secretion of splenic CD4+ $T$ cells in septic rats. (4A) The proliferative activity of CD4+ $T$ cells was measured by CCK8 assay and quantified by ELISA plate reader at $450 \mathrm{~nm}$. (4B) Levels of IL-2, IL-4, and IFN- $\gamma$ in culture supernatants were determined by ELISA kits. (4C) The mRNA expressions of IL-2, IL-4, and IFN- $\gamma$ in CD4+ $T$ cells were quantified by quantitative real-time PCR ( $=6$, ${ }^{*} P<0.01$ as compared with the sham groups; $\# P<0.01$ as compared with the sepsis groups; $* * 0.01$ as compared with other dose groups). 

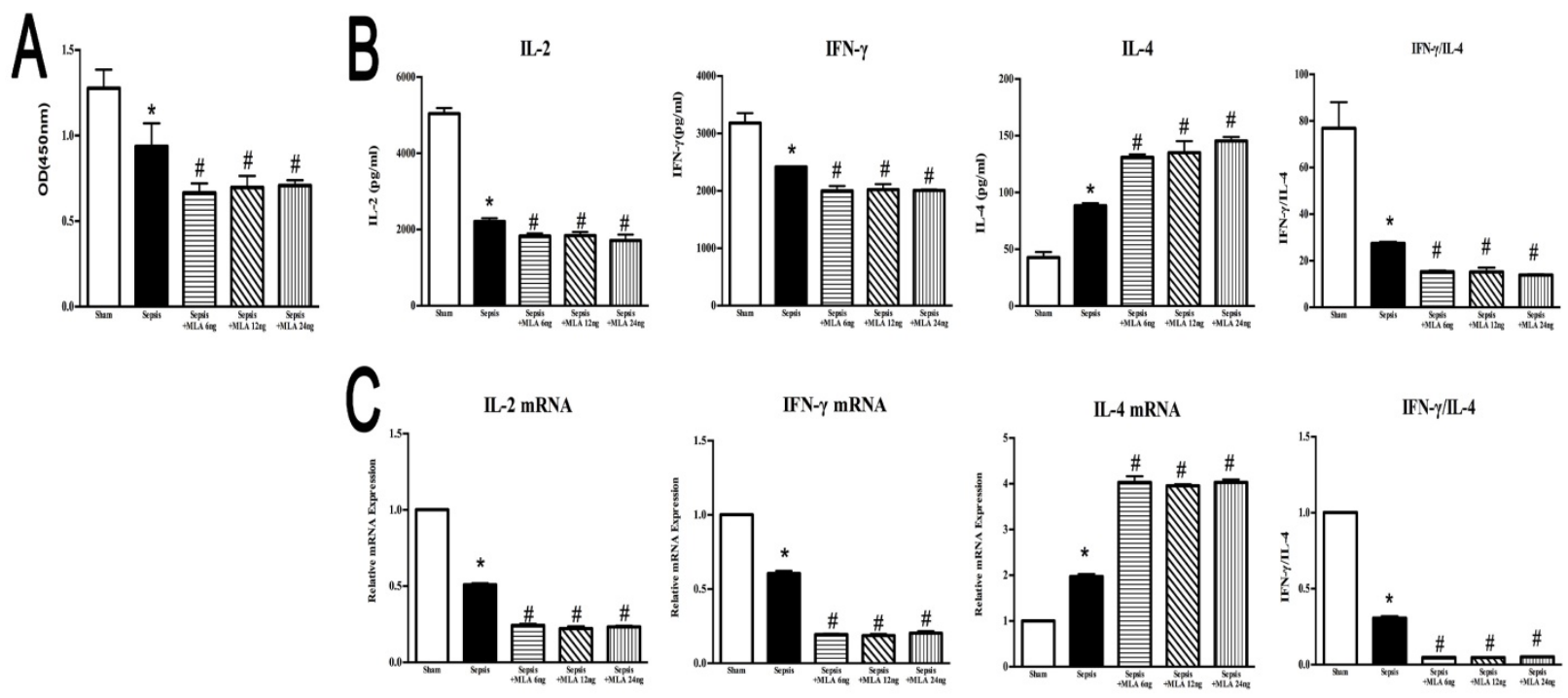

Figure 5. Effects of treatment with MLA on proliferation and secretion of splenic CD4+ $T$ cells in rats following septic challenge. (5A) The proliferative activity of $\mathrm{CD}^{+} \mathrm{T}$ cells was measured by CCK8 assay and quantified by ELISA plate reader at $450 \mathrm{~nm}$. (5B) Levels of IL-2, IL-4, and IFN- $\gamma$ in culture supernatants were determined by ELISA kits. (5C) The mRNA expressions of IL-2, IL-4, and IFN- $\gamma$ in CD4+ $T$ cells were quantified by quantitative real-time PCR ( $n=6$, ${ }^{*} P<0.01$ as compared with the sham groups; $\# P<0.01$ as compared with the sepsis groups).
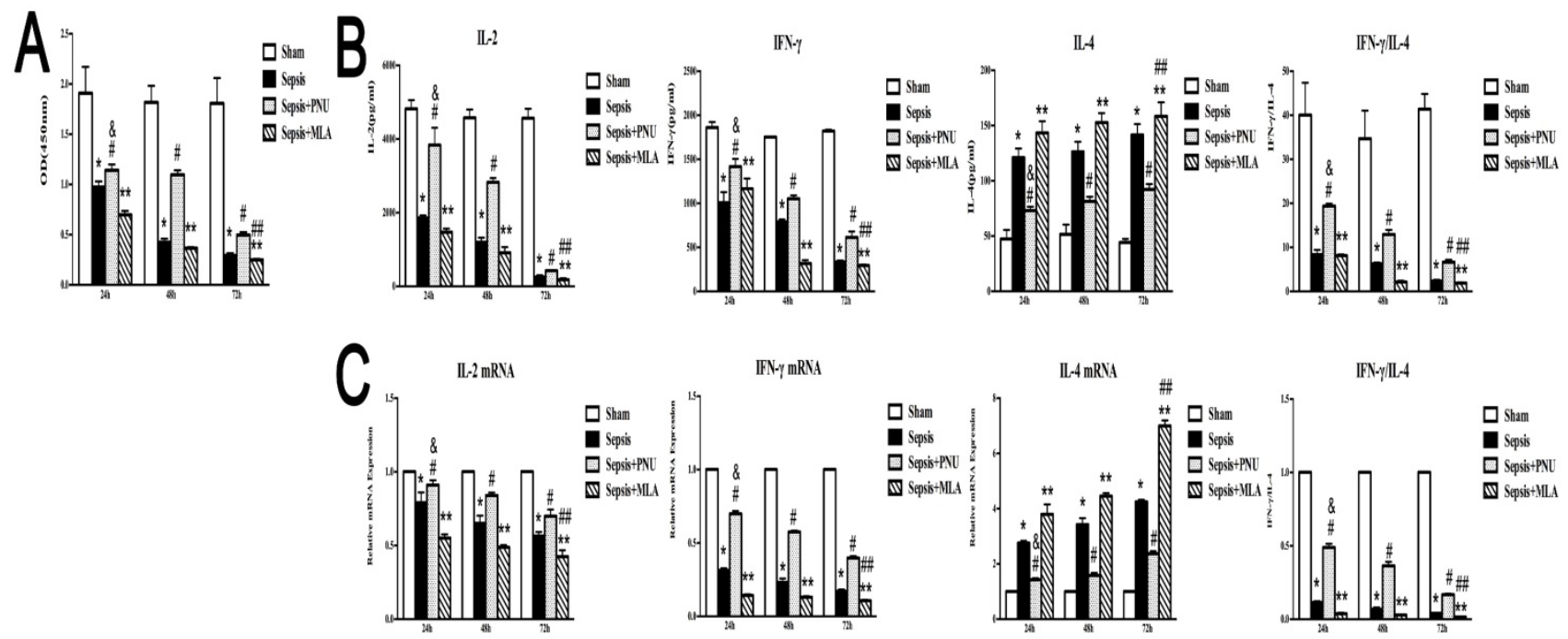

Figure 6. The time-dependent response of PNU282987 and MLA on the immune function of splenic CD4+ $T$ cells in rats following septic challenge. (6A) The proliferative activity of $C D 4^{+} T$ cells was measured by CCK8 assay and compared among different time points after CLP surgery. (6B) Levels of IL-2, IL-4, and IFN-Y in culture supernatants were measured by ELISA kits. (6C) The mRNA expressions of IL-2, IL-4, and IFN- $\gamma$ in CD4+ $T$ cells were quantified by quantitative real-time PCR $\left(n=6,{ }^{*} P<0.01\right.$ as compared with the sham groups at the identical time points; $\# P<0.01$ as compared with the sepsis groups at the identical time points; $* * 0.01$ as compared with the sepsis groups at the identical time points; $\& P<0.01$ as compared with different time points with PNU282987 administration; \#P $<0.01$ as compared with different time points with MLA administration).

Current study demonstrated that activation of central a7nAchR markedly ameliorated multiple organ injury, including heart, liver, lungs, and kidneys, and improved survival rates of septic rats. These organs all displayed severe injury of histomorphology compromised by severe sepsis, accompanied with notable elevation of corresponding functional markers [25, 26]. However, these abnormalities were obviously reversed by ICV injection of PNU282987 in a dose-independent pattern. To our knowledge, this was the first report that illustrated the effect of central a7nAchR modulation on peripheral immune response and organ dysfunction under septic exposure. With the dose-independent effects of PNU282987, it was legitimate since $a 7 n A c h R$ acted as an ion channel, and was prone to enter the desensitized state when subjected to overdosed stimulations [27, 28]. Therefore, maintaining the functional stability of a7nAchR might be a prerequisite for effective 
treatment of septic complication. We further evaluated the organ damage after brain $\mathrm{a} 7 \mathrm{nAchR}$ was inhibited by ICV injection of MLA. The heart, liver, lungs, and kidneys, apparently, revealed more severe injury than those in the sepsis groups, including deteriorated abnormalities of histomorphology, and markedly elevated levels of organ functional parameters, which suggested that rats with suppressed activity of central $a 7 \mathrm{nAchR}$ were more susceptible to septic challenge. This is also identical with the tendency that has been observed in a7nAChR-/- mice, as reported previously [29]. In the present study, the time-dependent effects of PNU282987 were evaluated, and they revealed significant protection against lethal sepsis at $24 \mathrm{~h}, 48$ $h$, and $72 \mathrm{~h}$ post CLP surgery, as rats at these time points underwent serious organ injury and developed notable mortality over the course of sepsis. Thus, central a7nAChR might be a potential therapeutic target for sepsis by alleviating multiple organ injury.

Immunosuppression is critically involved in the development of MODS and poor outcome secondary to septic episode [30]. The a7nAChR on immune cells has been recognized with potent anti-inflammatory and immunomodulatory capacities, and exerts beneficial effects by attenuating organ injury and improving survival of septic animals [22, 29, 31]. Nevertheless, the role of central a7nAChR on periphery cell-mediated immunity has not been reported in the setting of sepsis. Herein, we found a significantly suppressed function of splenic $\mathrm{CD} 4^{+} \mathrm{T}$ cells owing to sepsis, as evidenced by decreased proliferative activity and lowered expressions of IL-2. Meanwhile, expressions of IFN- $\gamma$ and IL-4 were presented with aberrant ratio that indicated the dominance of Th2 polarization in septic rats. Activation of central a7nAChR significantly reversed the immunosuppressive state of $\mathrm{CD}^{+} \mathrm{T}$ lymphocytes at different time points post CLP surgery, which was independent of dosage. While exaggerated dysfunction of $\mathrm{CD} 4^{+} \mathrm{T}$ cells was noted in MLA treated septic rats. These results indicated a certain effect of central $\mathrm{a} 7 \mathrm{nAChR}$ on the recovery and reconstruction of immune function in severe septic models, which showed a distinct deviation between central and periphery a7nAChR. Indeed, the effects of periphery a7nAChR stimulation were mainly reported in alleviating the excessive inflammatory response after the onset of sepsis, involving reduced production of pro-inflammatory mediators and inhibited activation of immune cells [32,33]. Likely, increased vagal activity was documented as a major contributor for immune paralysis in patients with traumatic brain injury, suggesting that the uncontrolled activity of periphery a7nAChR might be a potential cause for sepsis induced immunosuppression [34, 35]. From the current experiments, however, central a7nAChR presented with protective effects against sepsis lethality and immunosuppression after activated via ICV injection of PNU282987, as mentioned above. In addition to different distribution, this discrepancy might also result from the different severity of septic condition induced by CLP operation, as established with outward signs of multiple organ dysfunction, immunosuppressive state together with high mortality rate in our observations. Actually, PNU282987 and MLA that were used as agonist and antagonist for $\alpha 7 \mathrm{nAChR}$ respectively were both small molecules, and could pass through the blood brain barrier to impact both central and periphery a7nAChR. However, the doses differed greatly between the two systems. Taking PNU282987 as an example, its molecular weight was about $264.7 \mathrm{~g} / \mathrm{mol}$, along with 2.4 for xLogP3-AA. Intraperitoneal administration of PNU282987 was reportedly beneficial for neurodegenerative disease and sepsis by attenuating local and system inflammatory response with the doses at $76 \mu \mathrm{g}$ to $600 \mu \mathrm{g}$ per rat, which was much higher than those used in our study $(0.25 \mu \mathrm{g}, 0.8$ $\mu \mathrm{g}$, and $2.56 \mu \mathrm{g}$ per rat). Obviously, the doses of PNU282987 was inadequate to control the systematic inflammation when manipulated by ICV injection, which was equally applied to MLA in the present study. Given that central muscarine receptor has been identified as the primary receptor for manipulating the CAP activation [36], cerebral a7nAChR might modulate the activity of CAP in an indirect way, which needs to be further investigated.

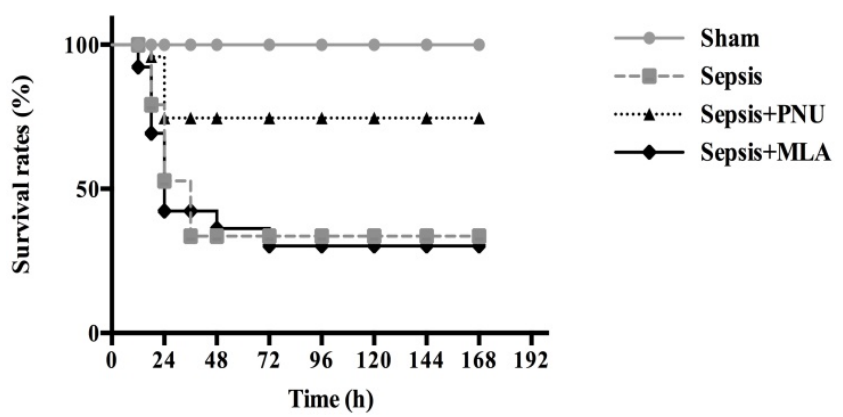

Figure 7. Effects of treatment with PNU282987 and MLA on the survival rates of septic rats. The survival rates were recorded at $12 \mathrm{~h}, 24 \mathrm{~h}, 36 \mathrm{~h}, 48 \mathrm{~h}, 72 \mathrm{~h}, 96$ $\mathrm{h}, 120 \mathrm{~h}, 144 \mathrm{~h}$, and $168 \mathrm{~h}$ post CLP operation, respectively. ICV injection of PNU282987 significantly increased the survival rate of septic rats in comparison to that in the sepsis group $(n=26,80 \%$ vs. $40 \%, P<0.05)$. No remarkable difference was found between the sepsis with MLA injection group and the sepsis group.

The present study provided the first evidence that activation of central $\mathrm{a} 7 \mathrm{nAchR}$ appeared to be beneficial for multiple organs dysfunction and immunosuppression under severe abdominal infection. As one of the major effectors for the 
neuro-endocrine immune network, the distinct role of central a7nAchR would promote new insights into the pathogenesis of sepsis and share a potential therapeutic target for ameliorating organ injury and modulating immune response. Thus, further researches that expect to explore specific mechanism underlying $\alpha 7 \mathrm{nAchR}$ in the treatment of sepsis should cover both the central and the periphery a7nAchR, as the brain $a 7 n A c h R$ might be a promising therapeutic target in improving the prognosis of septic cases. Nonetheless, our study only described the protective effects of central a7nAchR on sepsis induced multiple organ injury and abnormal immune response, its precise mechanism remained to be clarified. Additionally, we have identified the role of central a7nAchR only by using selective agonists and inhibitors, but evidences on rats with $a 7 n A c h R$ gene knockdown were absent. It would undoubtedly provide stronger evidences for illustrating the effects of brain a7nAchR on sepsis induced multiple organ injury and immunosuppression when the evidences of $\mathrm{a} 7 \mathrm{nAchR}$ gene knockout rats were added. With regard to the pathogenesis of host immune dissonance, we mainly focused on the adaptive immune system, the impact of cerebral $\alpha 7 n A c h R$ on the innate immune system should be further elucidated. In addition, clarifying the interaction between brain $a 7 n A c h R$ and CAP might be helpful to improve the understanding of $\alpha 7 \mathrm{nAchR}$ function associated with septic complications.

\section{Conclusion}

In summary, these findings delineated a protective effect of central $\alpha 7 n A c h R$ on reversing immunosuppression and attenuating multiple organ dysfunction, followed by significantly improved outcome of septic rats. While these signs revealed remarkable deterioration after inhibition of cerebral $\alpha 7 n A c h R$. Therefore, the $\alpha 7 n A c h R$ might be a promising target for the treatment of sepsis in the way of central activation.

\section{Abbreviations}

a7nAchR: Alpha 7 nicotinic acetylcholine receptor; CAP: cholinergic anti-inflammatory pathway; CLP: cecal ligation and puncture; MLA: methyllycaconitine; HE: Hematoxylin-eosin; W/D: wet-to-dry weight ratio; MPO: myeloperoxidase; IL-2: interleukin 2; IL-4: interleukin 4; IFN- $\gamma$ : interferon- $\gamma$; PCR: polymerase chain reaction; ELISA: enzymelinked immunosorbent assay; Th cells: helper T cell; ICU: intensive care units; MODS: multiple organ dysfunction syndrome; TNF-a: tumor necrosis factor-a; HMGB1: high mobility group box-1 protein; ICV: Intracerebroventricular; CK: creatine kinase;
CK-MB: creatine kinase-MB; AST: aspartate aminotransferase; ALT: alanine aminotransferase; CG: cholylglycine; BUN: blood urea nitrogen; $\mathrm{Cr}$ : creatinine; CCK8: Cell counting kit-8; Con A: concanavalin A.

\section{Acknowledgements}

This work was supported by grants from the National Natural Science Foundation (Nos. 81730057, 81501664, 81372054), and the National Key Research and Development Program of China (No. 2017YFC1103302).

\section{Authors' contributions}

YMY conceived of the project and its study design, participated in drafting and revising the manuscript. CR and XHL helped to design the experiments and wrote this paper, contributed to the data acquisition, analyzed and interpreted the data. XHL and SBW performed the experiments, interpreted and analyzed the data, revised the manuscript. CR, XHL and SBW contributed equally to this work. ND and LXW helped to construct animal models and perform HE staining, analyzed image and data. YW contributed to ELISA experiments and data interpretation.

\section{Competing Interests}

The authors have declared that no competing interest exists.

\section{References}

1. Ward PA. New approaches to the study of sepsis. EMBO Mol Med. 2012;4:1234-43.

2. Martin GS, Mannino DM, Moss M. The effect of age on the development and outcome of adult sepsis. Crit Care Med. 2006;34:15-21.

3. Hotchkiss RS, Monneret G, Payen D. Sepsis-induced immunosuppression: from cellular dysfunctions to immunotherapy. Nat Rev Immunol. 2013;13:862-74.

4. Deng D, Li X, Liu C, et al. Systematic investigation on the turning point of over-inflammation to immunosuppression in CLP mice model and their characteristics. Int Immunopharmacol. 2017;42:49-58.

5. Antonakos N, Tsaganos T, Oberle V, et al. Decreased cytokine production by mononuclear cells after severe gram-negative infections: early clinical signs and association with final outcome. Crit Care. 2017;21:48.

6. Tracey KJ. The inflammatory reflex. Nature. 2002;420:853-9.

7. Pavlov VA, Ochani M, Yang LH, et al. Selective alpha7-nicotinic acetylcholine receptor agonist GTS-21 improves survival in murine endotoxemia and severe sepsis. Crit Care Med. 2007;35:1139-44.

8. Kim TH, Kim SJ, Lee SM. Stimulation of the alpha7 nicotinic acetylcholine receptor protects against sepsis by inhibiting Toll-like receptor via phosphoinositide 3-kinase activation. J Infect Dis. 2014;209:1668-77.

9. Andersen N, Corradi J, Sine SM, et al. Stoichiometry for activation of neuronal alpha7 nicotinic receptors. Proc Natl Acad Sci U S A. 2013;110:20819-24.

10. Wallace TL, Porter RH. Targeting the nicotinic alpha7 acetylcholine receptor to enhance cognition in disease. Biochem Pharmacol. 2011;82:891-903.

11. Rousseau SJ, Jones IW, Pullar IA, et al. Presynaptic alpha7 and non-alpha7 nicotinic acetylcholine receptors modulate $[3 \mathrm{H}] \mathrm{d}$-aspartate release from rat frontal cortex in vitro. Neuropharmacology. 2005;49:59-72.

12. Livingstone PD, Wonnacott $\mathrm{S}$. Nicotinic acetylcholine receptors and the ascending dopamine pathways. Biochem Pharmacol. 2009;78:744-55.

13. Kong FJ, Ma LL, Zhang HH, et al. Alpha 7 nicotinic acetylcholine receptor agonist GTS-21 mitigates isoflurane-induced cognitive impairment in aged rats. J Surg Res. 2015;194:255-61.

14. Hu S, Cui W, Mak S, et al. Substantial Neuroprotective and Neurite Outgrowth-Promoting Activities by Bis(propyl)-cognitin via the Activation of Alpha7-nAChR, a Promising Anti-Alzheimer's Dimer. ACS Chem Neurosci. 2015;6:1536-45. 
15. Sussman ES, Kellner CP, McDowell MM, et al. Alpha-7 nicotinic acetylcholine receptor agonists in intracerebral hemorrhage: an evaluation of the current evidence for a novel therapeutic agent. Neurosurg Focus. 2013;34:E10.

16. Pavlov VA, Tracey KJ. Controlling inflammation: the cholinergic anti-inflammatory pathway. Biochem Soc Trans. 2006;34:1037-40.

17. Rhodes A, Evans LE, Alhazzani W, et al. Surviving Sepsis Campaign: International Guidelines for Management of Sepsis and Septic Shock: 2016. Crit Care Med. 2017;45:486-552.

18. Hotchkiss RS, Monneret G, Payen D. Immunosuppression in sepsis: a novel understanding of the disorder and a new therapeutic approach. Lancet Infect Dis. 2013;13:260-8.

19. Russell JA. Management of sepsis. N Engl J Med. 2006;355:1699-713.

20. Andersson U, Tracey KJ. Neural reflexes in inflammation and immunity. J Exp Med. 2012;209:1057-68.

21. Bencherif M, Lippiello PM, Lucas R, et al. Alpha7 nicotinic receptors as novel therapeutic targets for inflammation-based diseases. Cell Mol Life Sci. 2011;68:931-49.

22. Tsoyi K, Jang HJ, Kim JW, et al. Stimulation of alpha7 nicotinic acetylcholine receptor by nicotine attenuates inflammatory response in macrophages and improves survival in experimental model of sepsis through heme oxygenase-1 induction. Antioxid Redox Signal. 2011;14:2057-70.

23. Fujii T, Mashimo M, Moriwaki $Y$, et al. Expression and Function of the Cholinergic System in Immune Cells. Front Immunol. 2017;8:1085.

24. Beinat $C$, Banister SD, Herrera $M$, et al. The therapeutic potential of alpha7 nicotinic acetylcholine receptor (alpha7 $\mathrm{nAChR}$ ) agonists for the treatment of the cognitive deficits associated with schizophrenia. CNS Drugs. 2015;29:529-42.

25. Vincent JL, Sakr Y, Sprung CL, et al. Sepsis in European intensive care units: results of the SOAP study. Crit Care Med. 2006;34:344-53.

26. Gotts JE, Matthay MA. Sepsis: pathophysiology and clinical management. BMJ. 2016;353:i1585.

27. Uteshev VV. alpha7 nicotinic $\mathrm{ACh}$ receptors as a ligand-gated source of $\mathrm{Ca}(2+)$ ions: the search for a Ca(2+) optimum. Adv Exp Med Biol. 2012;740:603-38

28. Zhang J, Xue F, Whiteaker $P$, et al. Desensitization of alpha7 nicotinic receptor is governed by coupling strength relative to gate tightness. J Biol Chem. 2011;286:25331-40

29. Su X, Matthay MA, Malik AB. Requisite role of the cholinergic alpha7 nicotinic acetylcholine receptor pathway in suppressing Gram-negative sepsis-induced acute lung inflammatory injury. J Immunol. 2010;184:401-10.

30. Boomer JS, To K, Chang KC, et al. Immunosuppression in patients who die of sepsis and multiple organ failure. Jama. 2011;306:2594-605.

31. Sitapara RA, Antoine DJ, Sharma L, et al. The alpha7 nicotinic acetylcholine receptor agonist GTS-21 improves bacterial clearance in mice by restoring hyperoxia-compromised macrophage function. Mol Med. 2014;20:238-47.

32. Nullens S, Staessens M, Peleman C, et al. Effect of Gts-21, an Alpha7 Nicotinic Acetylcholine Receptor Agonist, on Clp-Induced Inflammatory, Gastrointestinal Motility, and Colonic Permeability Changes in Mice. Shock. 2016;45:450-9.

33. Parrish WR, Rosas-Ballina M, Gallowitsch-Puerta M, et al. Modulation of TNF release by choline requires alpha7 subunit nicotinic acetylcholine receptor-mediated signaling. Mol Med. 2008;14:567-74.

34. Hall S, Kumaria A, Belli A. The role of vagus nerve overactivity in the increased incidence of pneumonia following traumatic brain injury. Br J Neurosurg. 2014;28:181-6.

35. Kox M, Pompe JC, Pickkers P, et al. Increased vagal tone accounts for the observed immune paralysis in patients with traumatic brain injury. Neurology. 2008;70:480-5.

36. Pavlov VA, Ochani $\mathrm{M}$, Gallowitsch-Puerta $\mathrm{M}$, et al Central muscarinic cholinergic regulation of the systemic inflammatory response during endotoxemia. Proc Natl Acad Sci U S A. 2006;103:5219-23. 